\title{
Squamous Metaplasia of the Stomach Associated with Lymphoma Infiltration
}

\author{
Masaya Iwamuro ${ }^{1}$, Nobuharu Fujii ${ }^{2}$, Takehiro Tanaka ${ }^{3}$, Hiromitsu Kanzaki ${ }^{1}$, Seiji Kawano ${ }^{1}$, \\ Yoshiro Kawahara ${ }^{4}$ and Hiroyuki Okada ${ }^{1}$
}

\begin{abstract}
:
We herein report a patient who presented with follicular lymphoma. Although the stomach was initially intact, mucosal redness and multiple erosions appeared in the gastric body owing to infiltration of the follicular lymphoma cells. Subsequently, a slightly depressed, white area lacking gastric mucosal structure was detected in the lesser curvature of the gastric cardia and body, where lymphoma cell infiltration was also pathologically observed beneath the stratified squamous epithelium. This case indicated that, although infrequent, prolonged mucosal injury owing to lymphoma infiltration can cause squamous metaplasia in the stomach.
\end{abstract}

Key words: esophagogastroduodenoscopy, squamous metaplasia, gastrointestinal lymphoma

(Intern Med 60: 2229-2234, 2021)

(DOI: 10.2169/internalmedicine.6271-20)

\section{Introduction}

Metaplasia refers to the histological transformation of one differentiated cell type to another differentiated cell type. Metaplastic lesions are often observed during esophagogastroduodenoscopy, such as changes from esophageal stratified squamous epithelium to simple columnar epithelium, known as Barrett's esophagus, from gastric epithelium to epithelium resembling the small intestines, and from duodenal mucosa to gastric-type mucus-secreting cells (1-3). In contrast, the development of squamous metaplasia is uncommon in the stomach. Thus, sequential endoscopic images of gastric squamous metaplasia have rarely been reported.

We herein report a case of follicular lymphoma that transitioned into squamous metaplasia of the stomach in association with lymphoma infiltration. Endoscopic images before and after the appearance of squamous metaplasia are also shown.

\section{Case Report}

A 45-year-old Japanese man underwent esophagogastroduodenoscopy during his annual screening. It revealed whitish granular elevations in the duodenum (Fig. 1A), and an endoscopic biopsy of the duodenal lesion showed infiltration of follicular lymphoma cells. Atrophic gastritis was observed, but there was no lymphoma involvement in the stomach (Fig. 1B). Ileal lesions were also identified during colonoscopy. In addition, the uptake of tracer was noted in the mesenteric lymph node on positron emission tomography (Fig. 1C). Bone marrow aspirate and biopsy specimens revealed no lymphoma cells in the bone marrow. At this point, his hemoglobin was $15.2 \mathrm{~g} / \mathrm{dL}$ and lactate dehydrogenase was $148 \mathrm{U} / \mathrm{L}$ (normal range: 120-240 U/L). Thus, a diagnosis of grade 1, stage $\mathrm{II}_{1}$ follicular lymphoma involving the duodenum, ileum, and mesenteric lymph node was made.

The patient was classified as low risk according to the Follicular Lymphoma International Prognostic Index (4). No treatment was initiated due to the absence of lymphoma-

\footnotetext{
${ }^{1}$ Department of Gastroenterology and Hepatology, Okayama University Graduate School of Medicine, Dentistry, and Pharmaceutical Sciences, Japan, ${ }^{2}$ Department of Hematology and Oncology, Okayama University Graduate School of Medicine, Dentistry and Pharmaceutical Sciences, Japan, ${ }^{3}$ Department of Pathology, Okayama University Hospital, Japan and ${ }^{4}$ Department of Practical Gastrointestinal Endoscopy, Okayama University Hospital, Japan

Received: September 9, 2020; Accepted: December 28, 2020; Advance Publication by J-STAGE: February 22, 2021

Correspondence to Dr. Masaya Iwamuro, iwamuromasaya@yahoo.co.jp
} 

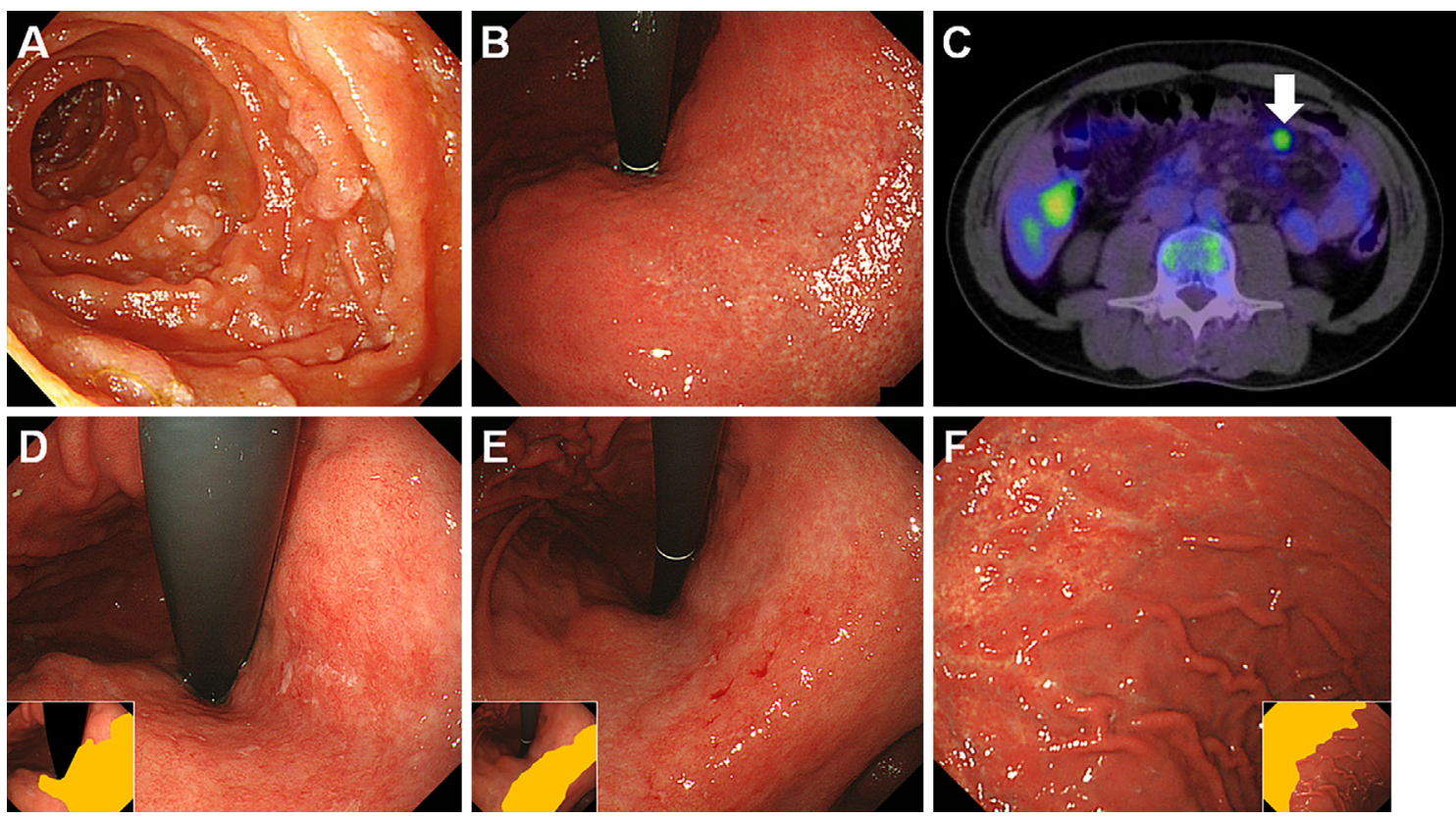

Figure 1. Endoscopic and positron emission tomography images. Esophagogastroduodenoscopy shows whitish granular elevations in the duodenum, which was diagnosed as follicular lymphoma (A) at the age of $\mathbf{4 5}$ years. Although atrophic gastritis is observed, there is no lymphoma involvement in the stomach (B). Mesenteric lymph node involvement is identified on positron emission tomography (C). Esophagogastroduodenoscopy at 48 years of age revealed mucosal redness and multiple erosions in the gastric body (D, E, yellow areas in the inserts) and whitish lesions in the greater curvature of the fornix ( $F$, yellow area in the insert).

associated symptoms. One year later, lymphoma progression with axillary and inguinal lymph node swelling was noted, but the patient was kept under active surveillance.

Despite the presence of atrophic gastritis, the stomach was initially intact; however, at 48 years of age, mucosal redness and multiple erosions appeared in the lesser curvature of the gastric body (Fig. 1D, E, yellow areas in the inserts), and whitish lesions appeared in the greater curvature of the fornix (Fig. 1F, yellow area in the insert). A biopsy of the erosions revealed infiltrations of follicular lymphoma cells. Initiation of treatment for gastric lymphoma was still waived since the patient was asymptomatic. Although Helicobacter pylori was detected in the stomach biopsy specimens obtained at 45 years of age, the patient was negative for serum $H$. pylori IgG antibody, and $H$. pylori was not detected in the biopsy specimens obtained at 49 years of age. Therefore, $H$. pylori was considered to have been spontaneously eradicated.

Esophagogastroduodenoscopy performed at 52 years of age showed progression of gastric involvement, with multiple small ulcers extending to the greater curvature of the gastric body (Fig. 2). Esophagogastroduodenoscopy performed at 54 years of age showed diffuse white mucosal lesions (Fig. 3A, yellow area in the insert) with spontaneous mucosal hemorrhaging (Fig. 3C). Histologically, small to medium-sized lymphoma cells existed extensively in the lesions (Fig. 4A, B). The lymphoma cells were positive for CD20 (Fig. 4C), CD10 (Fig. 4D), and BCL2 (Fig. 4E) but negative for CD3 (Fig. 4F). Furthermore, a slightly depressed, white area lacking gastric mucosal structure was observed in the lesser curvature of the gastric cardia and body (Fig. 3A, B, white areas in the inserts). Indigo carmine spraying emphasized the clear boundaries of the smooth surface area (Fig. 3D, white area in the insert), and a biopsy revealed lymphoma cell infiltration beneath the stratified squamous epithelium (Fig. 5). Thus, a diagnosis of squamous metaplasia of the stomach in association with lymphoma infiltration was made. The metaplastic lesion was contiguous with the esophageal squamous mucosa.

Positron emission tomography revealed an increased number of swollen axillar, mediastinal, mesenteric, and pelvic lymph nodes. Due to the spontaneous hemorrhaging from the gastric lymphoma lesions, he was treated with rituximab $\left(375 \mathrm{mg} / \mathrm{m}^{2}\right)$ intravenously once weekly for 4 weeks, which resulted in eradication of the follicular lymphoma cells in the stomach (Fig. 6A, B). However, the axillary and pelvic lymph node involvement was not decreased. Although the lymphoma cells in the stomach disappeared, squamous metaplasia of the stomach persisted (Fig. 6A, B, white area in the insert). On magnifying observation with narrow-band imaging, squamous metaplasia was observed as white mucosal lesions where gastric pits were absent (Fig. 6C). In contrast to the normal squamous epithelium of the esophagus, in the squamous metaplasia, the intra-papillary capillary loop was not observed. The patient was treated with rituximab every two to three months for two additional years as 

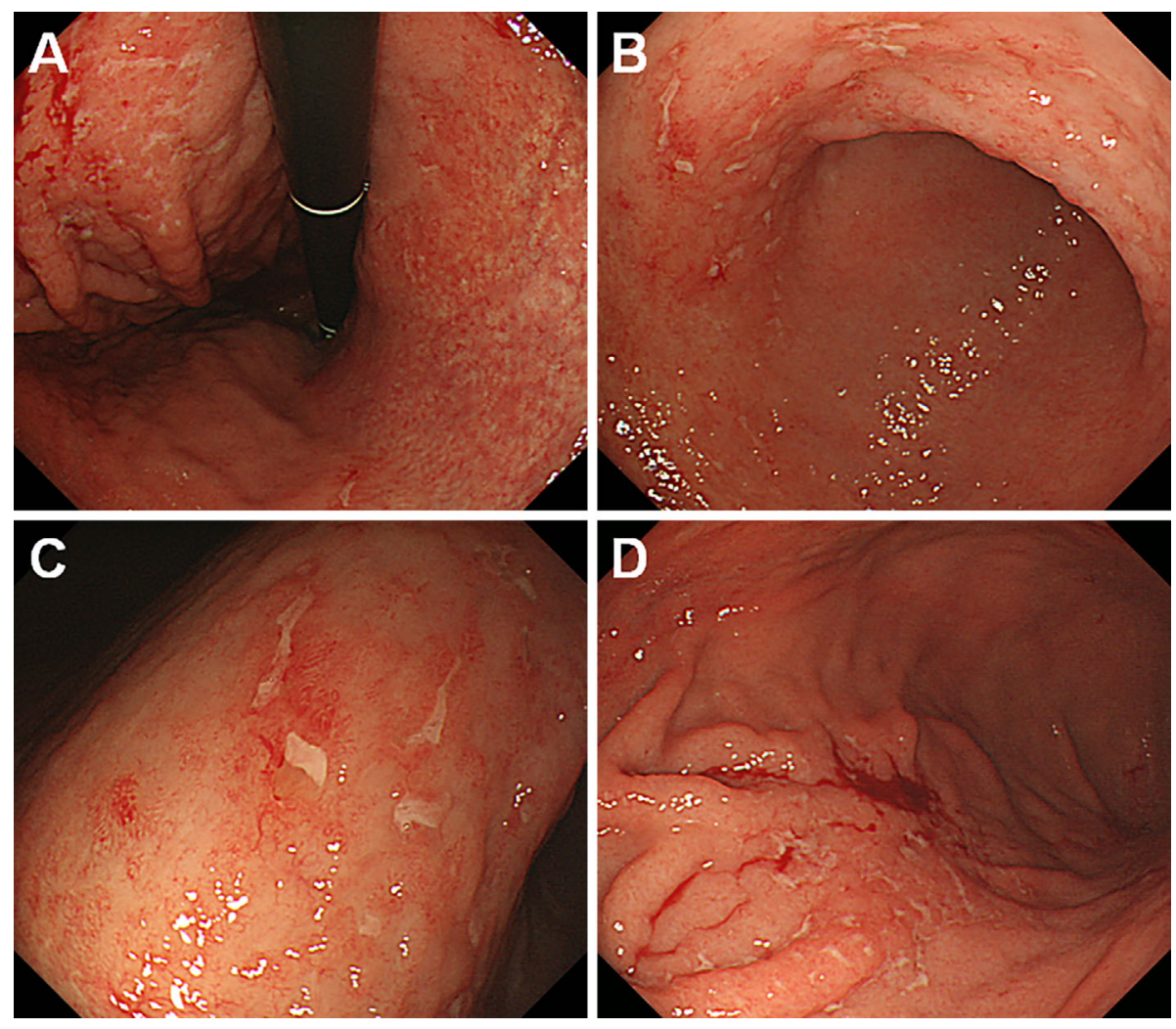

Figure 2. Esophagogastroduodenoscopy at 52 years of age. Esophagogastroduodenoscopy shows the progression of gastric involvement, with multiple small ulcers extending to the greater curvature of the gastric body.

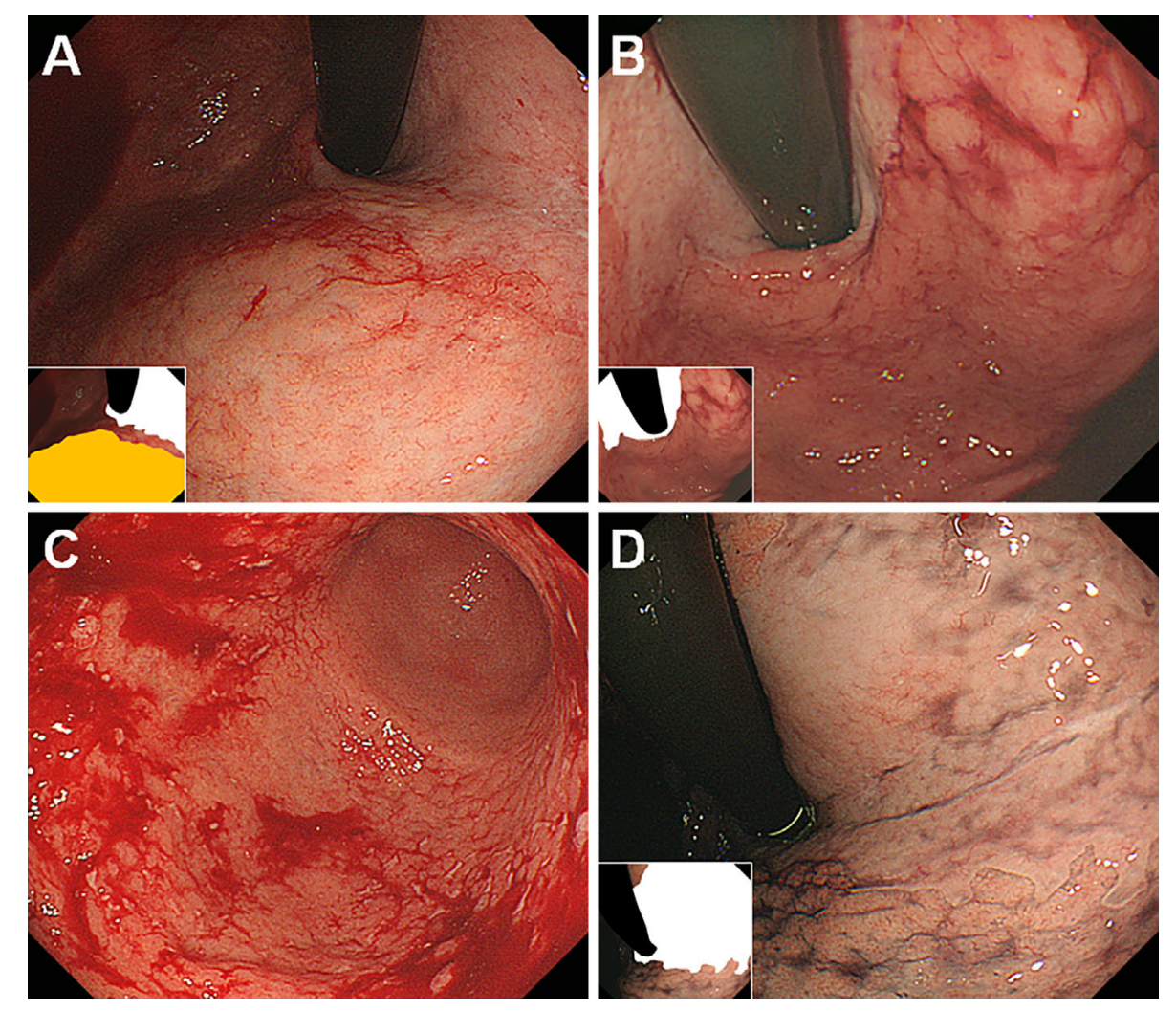

Figure 3. Endoscopic images at 54 years of age. Esophagogastroduodenoscopy shows the progression of gastric involvement, with diffuse white mucosal lesions (A, yellow area in the insert) and a slightly depressed, white area lacking gastric mucosal structures (A, B, white areas in the inserts). Spontaneous mucosal hemorrhaging is also observed $(C)$. Indigo carmine spraying emphasizes the clear boundaries of the smooth surface area $(\mathrm{D}$, white area in the insert is squamous metaplasia). 

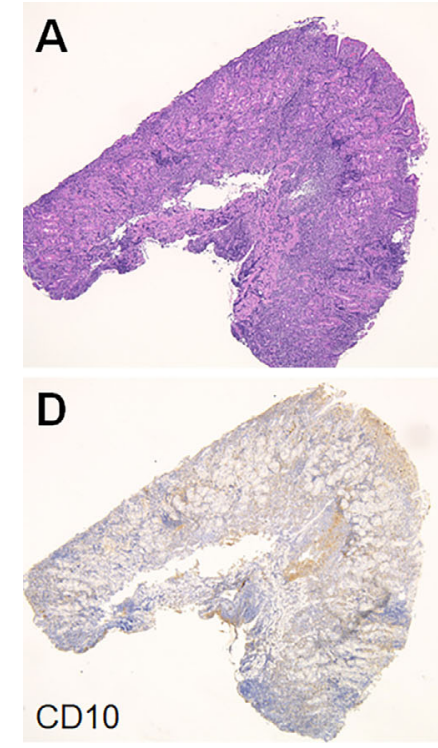
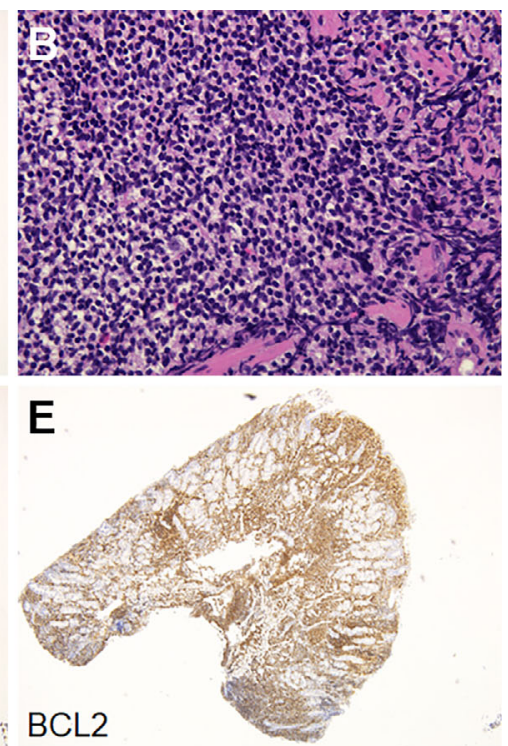

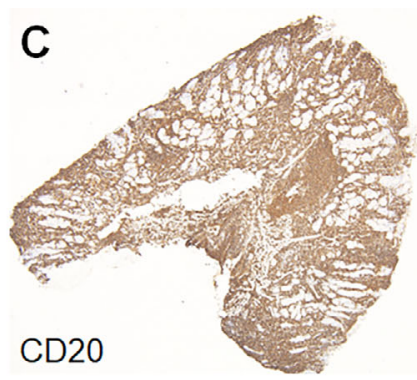

$\mathrm{F}$

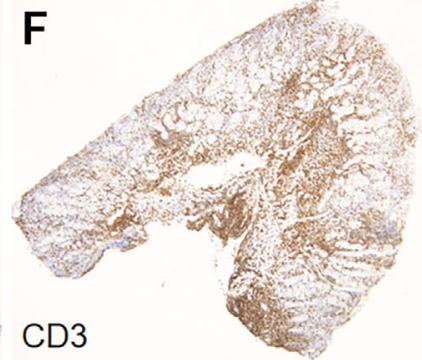

Figure 4. Pathological images. Small to medium-sized lymphoma cells exist extensively in the white mucosal lesions (A, B). The lymphoma cells are positive for CD20 (C), CD10 (D), and BCL2 (E) but are negative for $\mathrm{CD3}(\mathrm{F})$.
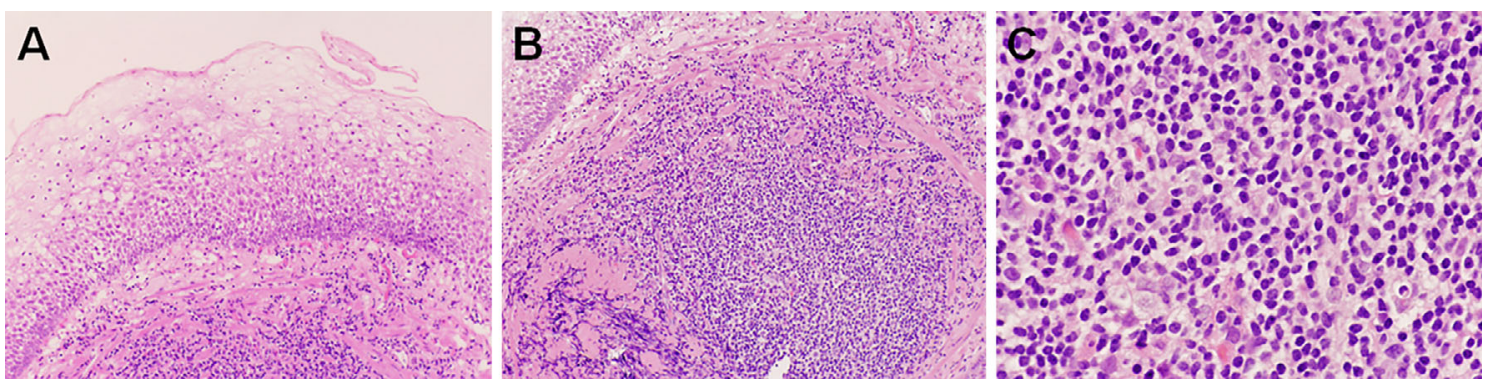

Figure 5. Pathological images of the squamous metaplasia. In the biopsy specimen of the slightly depressed, white area, lymphoma cell infiltration can be seen beneath the stratified squamous epithelium.
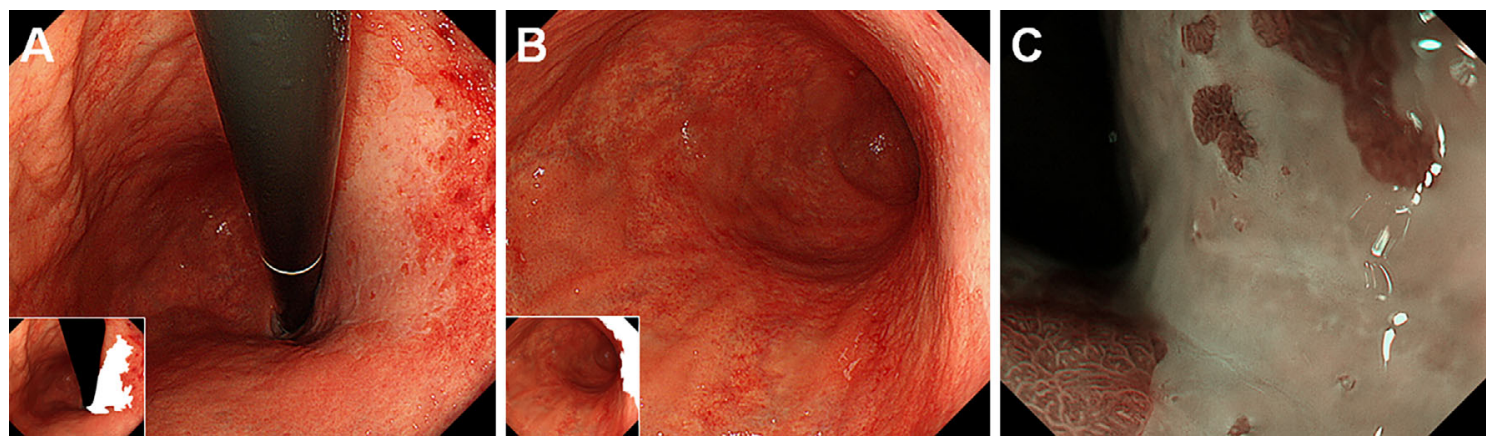

Figure 6. Endoscopic images after rituximab treatment. Although the follicular lymphoma cells disappeared (A, B), squamous metaplasia of the stomach persists (A, B, white areas in the insert). On magnifying observation with narrow-band imaging, squamous metaplasia is observed as white mucosal lesions (C).

maintenance therapy. At the last visit, the patient had retroperitoneal, pelvic, and inguinal lymphadenopathies, while gastric and duodenal involvement was absent. He was kept under active surveillance.

\section{Discussion}

In contrast to the infrequent observations of squamous 
metaplasia in the stomach, transition of the epithelium to squamous cells is more commonly seen in the uterine cervix, bladder, and airways (5). Such squamous metaplasia is considered to develop as a consequence of mechanical irritation and/or chronic inflammation. Similarly, gastric squamous metaplasia has previously been reported in patients with syphilitic gastritis $(6,7)$, gastric tuberculosis $(8)$, and corrosive gastritis (9). These cases indicate that squamous metaplasia develops in the stomach under chronic inflammation or transient massive mucosa damage. It is speculated that altered cellular differentiation occurs in the gastric stem cells during the regenerative process, constituting the initial source of squamous metaplasia (10).

To our knowledge, only two cases of gastric squamous metaplasia that developed in association with lymphoma lesions have been reported. In 1985, Callery et al. reported a case of squamous metaplasia and squamous cell carcinoma in the stomach (11). At 49 years of age, the patient underwent radiotherapy for lymphoma of the head and neck, which resulted in a complete response. However, the lymphoma recurred at the age of 52, showing splenomegaly, inguinal adenopathy, and diffuse intestinal involvement. He underwent chemotherapy with cyclophosphamide, vincristine, prednisone, and procarbazine, followed by maintenance treatment with chlorambucil. At the age of 55, a nodular mass emerged along the lesser curvature of the stomach, and a pathological diagnosis of squamous cell carcinoma coupled with squamous metaplasia in the gastric cardia was made based on the findings of the surgically resected specimen. The other patient was reported by Fujino et al. in a Japanese article in 1992 (12); he was 45 years old and was diagnosed with gastric diffuse large B-cell lymphoma. The gastric lymphoma was initially observed as an elevated lesion in the posterior wall of the gastric body. Subsequently, an ulcerative lesion emerged in the lesser curvature of the gastric body, which further transitioned to a whitish, slightly depressed area. Since radiological examinations revealed that the lymphoma lesion was localized in the stomach, he was treated with total gastrectomy and splenectomy. A postsurgical pathological analysis confirmed that the whitish area was squamous metaplasia. Lymphoma cells were also observed in the gastric body.

Although patients with squamous metaplasia have been reported in addition to the aforementioned two cases, to our knowledge, the detailed sequential endoscopic images shown here are the first obtained in the reporting of squamous metaplasia. The present patient had duodenal involvement, while the stomach was initially intact (Fig. 1B). Follicular lymphoma cell infiltration appeared in the lesser curvature of the gastric body, which showed multiple erosions and mucosal redness (Fig. 2). As the lymphoma progressed towards the entire stomach, a slightly depressed, white area emerged in the lesser curvature of the gastric cardia and body (Fig. 3), which was pathologically diagnosed as gastric metaplasia. These endoscopic images, together with the previous two reports on patients with lymphoma, indicate that squamous metaplasia can occur in the stomach during the progression of lymphoma.

Gastric squamous metaplasia is most frequently observed in the lesser curvature of the gastric cardia and body, as described in the present patient (13-16). These features support the hypothesis that squamous metaplasia arises from multipotent stem cells located in the esophagogastric junction (13). In contrast, the emergence of squamous metaplasia in other parts of the stomach has also been reported, including the antrum (17) and the prepylorus (18). Given that whitish lesions without glandular structures are the representative endoscopic characteristics of gastric squamous metaplasia, endoscopists should recognize these features for a prompt diagnosis.

Cases of squamous cell carcinoma in the stomach accompanied by squamous metaplasia have been previously reported $(9,11,16,19)$. Squamous metaplasia also occurs in the respiratory tree and urinary tract as a reactive response to chronic inflammation (20). In these organs, the induction of squamous differentiation is considered to perform a protective role by increasing the cell survival and tissue resilience (21). However, owing to its infrequency, whether or not gastric squamous metaplasia plays a protective role against lymphoma recurrence has not been clarified. In contrast, several authors hypothesized the involvement of a metaplasia-dysplasia-carcinoma sequence in patients with gastric squamous metaplasia. Therefore, careful endoscopic surveillance may be required for these patients (13).

Primary gastrointestinal follicular lymphoma most frequently affects the small intestine $(22,23)$, and in most patients, this disease is diagnosed by esophagogastroduodenoscopy as whitish small polypoid nodules in the duodenum, as observed in the present patient (Fig. 1A) $(23,24)$. Gastric involvement has been occasionally reported; its macroscopic features vary and can include protruding lesions (25); superficial, shallow depressed lesions (26); and multiple ulcers (Fig. 2). It is noteworthy that the present patient showed whitish lesions in the stomach (Fig. 3A). Therefore, although lymphoma lesions in the stomach generally present with various macroscopic features, the present case suggests that endoscopists need to consider the relevance of squamous metaplasia for whitish lesions involving the stomach that develop during the treatment of lymphoma.

In conclusion, we presented sequential endoscopic images of squamous metaplasia that developed from lymphoma in the stomach. This case indicates that, although infrequent, prolonged mucosal injury owing to lymphoma infiltration can cause squamous metaplasia in the stomach.

The authors state that they have no Conflict of Interest (COI).

\section{References}

1. Shah SC, Gawron AJ, Li D. Surveillance of gastric intestinal metaplasia. Am J Gastroenterol 115: 641-644, 2020.

2. Shah SC, Gawron AJ, Mustafa RA, Piazuelo MB. Histologic sub- 
typing of gastric intestinal metaplasia: overview and considerations for clinical practice. Gastroenterology 158: 745-750, 2020.

3. Dhaliwal L, Codipilly DC, Gandhi P, et al. Neoplasia detection rate (NDR) in Barrett's esophagus and its impact on missed dysplasia: results from a large population-based database. Clin Gastroenterol Hepatol. Forthcoming.

4. Solal-Céligny P, Roy P, Colombat $\mathrm{P}$, et al. Follicular lymphoma international prognostic index. Blood 104: 1258-1265, 2004.

5. Moshi JM, Hoogduin KJ, Ummelen M, et al. Switches of SOX17 and SOX2 expression in the development of squamous metaplasia and squamous intraepithelial lesions of the uterine cervix. Cancer Med 9: 6330-6343, 2020.

6. Singer HA. Leukoplakia of the stomach. Arch Pathol 9: 676-682, 1930.

7. Sailer S. Diffuse metaplastic gastritis in a patient with prolonged cahexia and macrocytic anemia. Arch Pathol 35: 739-743, 1943.

8. Watson GW, Flint ER, Stewart MJ. Hyperplastic tuberculosis of the stomah causing hour-glass deformity. With complete squamous metaplasia of the upper loculus. Br J Surg 24: 333-340, 1936.

9. Eaton H, Tennekoon GE. Squamous carcinoma of the stomach following corrosive acid burns. Br J Surg 59: 382-387, 1972.

10. Wood DA. Adenoacanthoma of the pyloric end of the stomach: a consideration of its histogenesis and a report of two cases. Arch Pathol 36: 177-189, 1943.

11. Callery CD, Sanders MM, Pratt S, Turnbull AD. Squamous cell carcinoma of the stomach: a study of four patients with comments on histogenesis. J Surg Oncol 29: 166-172, 1985.

12. Fujino K, Ichikukra T, Ikawa $H$, et al. A case report of malignant lymphoma of the stomach developing diffuse squamous metaplasia during follow-up. Gastroenterol Endosc 34: 1895-1899, 1992.

13. Takeda H, Nagashima R, Goto $T$, Shibata $Y$, Shinzawa $H$, Takahashi T. Endoscopic observation of squamous metaplasia of the stomach: a report of two cases. Endoscopy 32: 651-653, 2000.

14. Jeon MS, Kim GH, Park DY, et al. A case of squamous metaplasia of the stomach. Clin Endosc 46: 407-409, 2013.

15. Ahn S, Bae GE, Kim KM. Exuberant squamous metaplasia of the gastric mucosa in a patient with gastric adenocarcinoma. Diagn Pathol 10: 46, 2015.
16. Oono Y, Fu K, Nagahisa E, et al. Primary gastric squamous cell carcinoma in situ originating from gastric squamous metaplasia. Endoscopy 42 (Suppl): E290-E291, 2010.

17. Ishida S, Masuhara M, Mizumoto K, et al. Squamous metaplasia without atypia. Endoscopy 47 (Suppl): E550-E551, 2015.

18. Cho YS, Kim JS, Kim HK, et al. A squamous metaplasia in a gastric ulcer scar of the antrum. World J Gastroenterol 14: 12961298, 2008.

19. Vaughan WP, Straus FH 2nd, Paloyan D. Squamous carcinoma of the stomach after luetic linitis plastica. Gastroenterology 72: 945948, 1977.

20. Ishizumi $T$, McWilliams $A$, MacAulay $C$, Gazdar A, Lam S. Natural history of bronchial preinvasive lesions. Cancer Metastasis Rev 29: 5-14, 2010.

21. Dotto GP, Rustgi AK. Squamous cell cancers: a unified perspective on biology and genetics. Cancer Cell 29: 622-637, 2016.

22. Takata K, Okada H, Ohmiya N, et al. Primary gastrointestinal follicular lymphoma involving the duodenal second portion is a distinct entity: a multicenter, retrospective analysis in Japan. Cancer Sci 102: 1532-1536, 2011.

23. Iwamuro M, Kondo E, Takata K, Yoshino T, Okada H. Diagnosis of follicular lymphoma of the gastrointestinal tract: a better initial diagnostic workup. World J Gastroenterol 22: 1674-1683, 2016.

24. Yamamoto S, Nakase H, Yamashita K, et al. Gastrointestinal follicular lymphoma: review of the literature. J Gastroenterol 45: 370388,2010 .

25. Iwamuro M, Okada H, Takata K, et al. Diagnostic accuracy of endoscopic biopsies for the diagnosis of gastrointestinal follicular lymphoma: a clinicopathologic study of 48 patients. Ann Diagn Pathol 18: 99-103, 2014.

26. Iwamuro M, Imagawa A, Kobayashi N, et al. Synchronous adenocarcinoma and follicular lymphoma of the stomach. Intern Med 52: 907-912, 2013.

The Internal Medicine is an Open Access journal distributed under the Creative Commons Attribution-NonCommercial-NoDerivatives 4.0 International License. To view the details of this license, please visit (https://creativecommons.org/licenses/ by-nc-nd/4.0/).

(C) 2021 The Japanese Society of Internal Medicine

Intern Med 60: 2229-2234, 2021 